\title{
Final Report Technical Report \\ Kinetic Modeling of Damage Repair, Genome Instability, and Neoplastic Transformation
}

\author{
Grant DE-FG02-03ER63541
}

R.D. Stewart, P.I.

\section{Summary of Accomplishments}

Inducible repair and pathway interactions may fundamentally alter the shape of doseresponse curves because different mechanisms may be important under low- and high-dose exposure conditions. However, the significance of these phenomena for risk assessment purposes is an open question. To help advance our understanding of the mechanisms underlying the shapes of dose-response curves, this project developed new modeling tools to better link the induction and biological processing of DNA damage to higher-level biological endpoints, including cell killing, neoplastic transformation and cancer. The project is motivated by the hypothesis that a multiscale system of molecular and cellular models can be used to better define the shapes of dose-response curves for cancer induction. As a first step towards testing this hypothesis, we examined ways of constructing and linking together systems of minimally parameterized models to better link the induction of DNA damage to cell killing, neoplastic transformation and cancer. We developed new strategies to calibrate and test multi-scale models using information from different levels in the biological hierarchy and identified critical model inputs related to genomic instability, neoplastic transformation and cell killing.

In addition to exploring approaches for the construction and testing of multiscale biological simulations, we also developed and tested specific models for selected molecular and cellular processes. The project scope included (1) the development of new approaches to simulate the induction and base excision repair (BER) of DNA damage using Monte Carlo methods and (2) the integration of data from the Monte Carlo simulations with kinetic models for higher-level biological endpoints. We also developed models to aid in the analysis and interpretation of data from experimental assays, such as the pulsed-field gel electrophoresis (PFGE) assay used to quantity the amount of DNA damage caused by ionizing radiation.

Several computer programs developed as part of this project have been made freely available for educational, research and commercial purposes through the world wide web. Selected aspects of the project were reported in a series of 8 peer-reviewed publications and 12 presentations at DOE workshops, university seminars and national and international conferences. Additional details and key project findings are summarized below.

\section{Multiscale Modeling of Biological Systems}

The physical, chemical and biological processes that ultimately lead to cancer span at least ten orders of magnitude on the time scale $\left(<10^{-3} \mathrm{~s}\right.$ to $\left.>10^{7} \mathrm{~s}\right)$ and ten orders of magnitude on the spatial scale $\left(\sim 10^{-10} \mathrm{~m}\right.$ to $\left.1 \mathrm{~m}\right)$. Multi-stage carcinogenesis models, such as the two-mutation clonal expansion model, capture many of the putative key steps associated with the development of cancer, and they do so using as few adjustable parameters as possible. However, the desire to keep the number of adjustable model inputs to a minimum means that the effects of many molecular and cellular processes must be lumped together. That is, the details of the specific 
biological mechanisms of action involved in the development of cancer must be replaced by nonspecific (average) mechanisms that capture events and processes occurring over very large time and spatial scales. However, some in vitro experiments suggest that the risks of low dose radiation exposures may be larger or smaller than the risks expected using simple linear high-tolow-dose extrapolation procedures. Studies such as these indicate that we need to find practical strategies to introduce additional (more specific) biological details into the cancer modeling process.

Top-Down (TD) Cancer Modeling: One approach, the Top-Down (TD) approach, involves introducing additional compartments or mechanisms of action into standard multi-stage cancer models. The available animal and human cancer incidence data are typically used to calibrate and test the refined model. Because the TD modeling approach usually emphasizes simplicity (fewer adjustable parameters) over specificity, in vivo data are more useful for model calibration and testing purposes than in vitro data. However this understandable bias towards in vivo data imposes severe constraints on our ability to introduce additional biological detail into the cancer modeling process. For example, the mechanisms of action that can be justified from an analysis of cancer incidence data are usually far too simplistic to explain the results of many potentially significant in vitro experiments. The fundamental issue is that additional parameters must be introduced into the modeling process with each new refinement in the postulated biological mechanisms. As the number of adjustable parameters increases, a situation quickly arises in which several different combinations of model inputs all provide equally good fits to the available cancer incidence data, i.e., the so-called parameter identifiability issue. The ongoing lack of in vivo data to overcome parameter identifiability issues poses many challenges for biologically based cancer modeling.

Bottom-Up (BU) Cancer Modeling: An alternate approach, the Bottom-Up (BU) approach, begins by formulating relatively detailed mathematical models for specific molecular and cellular processes. For example, one model might capture key elements of the base excision repair (BER) pathway while another model relates the overall mutation rate to the onset of genomic instability and to the eventual neoplastic transformation of a cell or one of its progeny. As a first test of the postulated mechanisms of action, these lower-level models ("sub-models") are tested against measured data from in vitro systems. To model the emergent behavior (response) of a group of cells or a tissue, these sub-models are linked together to form a "supermodel.” The links (information pathways) among the sub-models represent specific hypotheses about the key events and processes involved in the development of cancer. As a second test of the postulated mechanisms, results from the supermodel can be compared to cancer incidence data.

The BU modeling approach provides a systematic method to balance the need for greater biological detail against the need for simplicity (fewer adjustable parameters). That is, each submodel uses a minimum number of adjustable parameters to explain the results from some particular set of in vitro experiments. The increase in biological specificity inherent in the BU approach usually requires a dramatic increase in the overall computational complexity of the model. The CPU-time needed to obtain numerical solutions as well as the accuracy and stability of these solutions can become important issues. As with the TD cancer modeling approach, parameter identifiability issues may arise during the calibration and testing of the sub-models and the supermodel. 
A central premise of the BU approach is that model inputs estimated from in vitro data should be used to simulate events and processes in vivo, unless of course in vivo data are available and suffice to estimate all model inputs. This approximation is invoked in order to keep the number of purely adjustable model inputs to a minimum while at the same time providing additional details about the presumed workings of specific biological mechanisms. The approximation that model inputs are the same in vitro and in vivo is reasonably well justified for endpoints such as DNA damage and, perhaps to a lesser extent, the outcome from a pathwayspecific DNA repair event (probability a cluster is correctly or incorrectly repaired). As we progress up through the biological hierarchy to processes such as the induction of apoptosis or the regulation of cell growth, the use of model inputs derived from in vitro data becomes increasingly questionable. However, setting some model inputs a priori to an approximate (in vitro) value may still be preferable to treating all of the model inputs as purely adjustable parameters.

\section{Induction of DNA Damage by Ionizing Radiation}

The passage of ionizing radiation through living organisms initiates physical and chemical processes that create clusters of damaged nucleotides within one or two turns of the DNA. These clusters are widely considered an important initiating event for the induction of other biological endpoints, including cell killing and neoplastic transformation. Monte Carlo simulations of the DNA damage formation process are a useful adjunct to experiments because they provide additional information about the spatial configuration of damage within a cluster.

We developed a fast and easy-to-implement Monte Carlo algorithm to simulate the local clustering of DNA lesions produced by ionizing electrons, protons and alpha particles with kinetic energies as high as GeV. This fast Monte Carlo Damage Simulation (MCDS) captures the major trends in the DNA damage spectrum predicted using detailed track-structure simulations, which suggests that the small-scale spatial distribution of DNA lesions is primarily determined by independent and purely stochastic events and processes. The MCDS model provides nucleotide-level information about the characteristics of various classes of damage produced by ionizing radiation, such as average number of lesions per DNA damage cluster and the cluster length in base pairs. Nucleotide-level maps of the clustered lesions formed by ionizing radiation are needed to better characterize the relative biological effectiveness (RBE) of high-energy protons and other light ions and as an input into to models that simulate the kinetics and ultimate outcome from the repair of DNA damage.

Additional information about the MCDS model and an executable version of the computer program (version 2.01, March 2006) has been made freely available for commercial, educational or research purposes through a website (http://rh.healthsciences.purdue.edu/mcds/).

\section{Models to Aid in the Analysis and Interpretation of Data from Pulsed-Field Gel Electrophoresis (PFGE) Assays}

Sample preparation procedures for the PFGE assay sometimes involve a lysis step at temperatures as high as $50^{\circ} \mathrm{C}$. During this warm-lysis procedure, multiply damaged sites containing heat-labile sites (HLS) can be converted into DSBs. Once formed, these DSBs cannot be distinguished from the DSBs formed directly by ionizing radiation. To aid in the analysis and interpretation of data from warm-lysis PFGE experiments, we develop a method to correct DSB 
estimates for the effects of HLS in warm-lysis protocols. The correction method uses a firstorder repair model to predict the number of HLS available for conversion into DSBs as a function of the time available for repair before initiating warm-lysis. A mathematical expression is derived to separate prompt DSBs from those formed through the artefactul conversion of HLS into DSBs. The proposed formalism only requires the specification of two adjustable parameters, both of which can be estimated from measured data.

Estimates of prompt DSB yields obtained by correcting warm-lysis data are in good agreement with estimates obtained using cold-lysis protocols, which do not include the effect of HLS. The retrospective analyses of two published datasets suggest that corrections for HLS have a substantial impact on DSB yields within the first 20 to $30 \mathrm{~min}$ after irradiation. Bi-exponential fits to the DSB data for CHO cells suggest that corrections for HLS reduce the half-time for fast DSB rejoining by about 15\%, whereas the half-time for the slow DSB rejoining only decreases by $4 \%$. The proposed formalism can be used to characterize trends and uncertainties in DSB rejoining kinetics associated with the artefactual conversion of HLS into DSBs. The retrospective application of the methodology to warm-lysis data enhances their relevance and usefulness for studies of DSB rejoining kinetics.

\section{Base Excision Repair of DNA Damage}

DNA is constantly damaged through endogenous processes and by exogenous agents, such as ionizing radiation. Base excision repair (BER) and nucleotide excision repair (NER) help maintain the stability of the genome by removing many different types of DNA damage. We developed a Monte Carlo excision repair (MCER) model that simulates key steps in the shortpatch and long-patch BER pathways and the NER pathway. The repair of both single and clustered damage, except double-strand breaks (DSBs), is simulated in the MCER model. Output from the model includes estimates of the probability that a cluster is repaired correctly, the fraction of the clusters converted into DSBs through the action of excision repair enzymes, the fraction of the clusters repaired with mutations, and the expected number of repair cycles needed to completely remove a clustered damage site. The quantitative implications of alternative hypotheses regarding the postulated repair mechanisms were investigated through a series of parameter sensitivity studies. These sensitivity studies are also used to help define the putative repair characteristics of clustered damage sites other than DSBs.

Clustered damage sites other than double-strand breaks (DSBs) have the potential to contribute to deleterious effects of ionizing radiation, such as cell killing and mutagenesis. We used the MCER model to simulate key steps in the BER and NER of DNA damage other than DSB and compared the results of these simulations to measured data for selected low-and highLET radiations. The Monte Carlo model reproduces experimental observations for the formation of enzymatic DSBs in Escherichia coli and cells of two Chinese hamster cell lines (V79 and xrs5). Comparisons of model predictions with experimental values for low-LET radiation suggest that an inhibition of DNA backbone incision at the sites of base damage by opposing strand breaks is active over longer distances between the damaged base and the strand break in hamster cells (8 bp) compared to E. coli (3 bp). Model estimates for the induction of point mutations in the human hypoxanthine guanine phosphoribosyl transferase (HPRT) gene by ionizing radiation are of the same order of magnitude as the measured mutation frequencies. Trends in the mutation frequency for low- and high-LET radiation are predicted correctly by the model. The agreement between selected experimental data sets and simulation results provides 
some confidence in postulated mechanisms for excision repair of DNA damage other than DSBs and suggests that the proposed Monte Carlo scheme is useful for predicting repair outcomes.

Additional information about the MCER model and an executable version of the computer program (version 2.01, March 2006) has been made freely available for commercial, educational or research purposes through a website (http://rh.healthsciences.purdue.edu/mcer/).

\section{Linking Double Strand Break Induction to Chromosomal Aberrations and Cell Killing}

To better link biochemical processing of the double strand break (DSB) to cell killing, a twolesion kinetic (TLK) model was developed and tested. In the TLK model, the family of all possible DSB is sub-divided into simple and complex DSB, and each kind of DSB may have its own repair characteristics. A unique aspect of the TLK model is that break-ends associated with both kinds of DSB are allowed to interact in pairwise fashion to form lethal and non-lethal chromosomal aberrations. To test the performance of the TLK model, we used non-linear optimization methods to calibrate the model based on CHO cell survival data for an extensive set of single-dose and split-dose exposure conditions. Then, some of the mechanisms of action postulated in the TLK were tested by comparing measured and predicted estimates of the initial DSB yield and the rate of DSB rejoining. TLK model predictions of CHO survival and the initial DSB yield and rejoining rate are all shown to be in good agreement with measured data. Studies suggest a yield of about $25 \mathrm{DSB} \mathrm{Gy}^{-1}$ cell $^{-1}$. About $20 \mathrm{DSB} \mathrm{Gy}^{-1}$ cell $^{-1}$ are rejoined quickly (15-minute repair half-time), and 4 to $6 \mathrm{DSB} \mathrm{Gy}^{-1}$ cell $^{-1}$ are rejoined very slowly (10 to 15 hour repair half-time). Both the slow- and fast-rejoining DSBs make a substantial contribution to the radiation killing of CHO cells. Although the TLK model provides a much more satisfactory formalism to relate biochemical processing of the DSB to cell killing than earlier kinetic models, some small differences among the measured and predicted CHO survival and DSB rejoining data suggest that additional factors and processes not considered in the present work may affect biochemical processing of the DSB and, hence, cell killing.

In a second study, we examined the theoretical and practical linkages between the TLK and linear-quadratic (LQ) model. We demonstrated that the LQ is an approximate time-integrated solution for the TLK and derived formulas linking DSB induction and repair parameters to intrinsic radiation sensitivity parameters used in the LQ model. We tested the equivalence of the TLK and LQ models using two extensive radiobiological datasets, one for CHO 10B2 cells and one for C3H 10T1/2 cells. Tests of model equivalence indicate that an LQ formula with two first-order repair terms is an excellent approximation to the TLK model.

\section{DNA Repair Effects in Carcinogenesis Models}

We developed a multistage cancer model that describes the putative rate-limiting steps in carcinogenesis. The model was used to investigate the potential impact on cumulative lung cancer incidence of the hormesis mechanisms suggested by Feinendegen and Pollycove. In the model, radiation and endogenous processes damage the DNA of target cells in the lung. Some fraction of the misrepaired or unrepaired DNA damage induces genomic instability and, ultimately, leads to the accumulation of malignant cells. The model explicitly accounts for cell birth and death processes, the clonal expansion of initiated cells, malignant conversion, and a lag period for tumor formation. Radioprotective mechanisms are incorporated into the model by postulating dose and dose-rate-dependent radical scavenging. The accuracy of DNA damage 
repair also depends on dose and dose rate. Sensitivity studies were conducted to identify critical model inputs and to help define the shapes of the cumulative lung cancer incidence curves that may arise when dose and doserate-dependent cellular defense mechanisms are incorporated into a multistage cancer model. For lung cancer, both linear no-threshold (LNT-), and non-LNTshaped responses can be obtained. If experiments demonstrate that the effects of DNA damage repair and radical scavenging are enhanced at least three-fold under low-dose conditions, our studies would support the existence of U-shaped responses. The overall fidelity of the DNA damage repair process may have a large impact on the cumulative incidence of lung cancer. The reported studies highlight the need to know whether or not (or to what extent) multiply damaged DNA sites are formed by endogenous processes. Model inputs that give rise to U-shaped responses are consistent with an effective cumulative lung cancer incidence threshold that may be as high as $300 \mathrm{mGy}$ (4 mGy per year for 75 years) for low-LET radiation.

\section{Summary of Project Deliverables}

\section{Number of Students Trained}

None

\section{Post-Docs Supported}

The project provided full support for 1 post-doctoral research associate (Vladimir A. Semenenko) and partial support for a second post-doctoral research associate (Ruwan K. Ratnayake).

\section{Journal Articles Published}

1. M. Guerrero, R.D. Stewart, J. Wang, and X.A. Li. Equivalence of the Linear-Quadratic and Two-Lesion Kinetic Models. Phys. Med. Biol. 47, 3197-3209 (2002).

2. V.A. Semenenko and R.D. Stewart. A fast Monte Carlo algorithm to simulate the spectrum of DNA damages formed by ionizing radiation. Radiat Res. 161(4), 451-457 (2004).

3. H. Schöllnberger, R.D. Stewart, R.E.J. Mitchel, and W. Hofmann. An examination of radiation hormesis mechanisms using a multi-stage carcinogenesis model. Nonlinearity in Biology, Toxicology and Medicine 2, 317-352 (2004).

4. H. Schöllnberger, R.D. Stewart, and R.E.J. Mitchel. Low-LET-induced radioprotective mechanisms within a two-stage cancer model. Dose-Response (formerly Nonlinearity in Biology, Toxicology and Medicine) 3, 508-518 (2005).

5. V.A. Semenenko, R.D. Stewart, E.J. Ackerman. Monte Carlo Simulation of Base and Nucleotide Excision Repair of Clustered DNA Damage Sites. I. Model Properties and Predicted Trends. Radiat. Res. 164, 180-193 (2005).

6. V.A. Semenenko and R.D. Stewart. Monte Carlo Simulation of Base and Nucleotide Excision Repair of Clustered DNA Damage Sites. II. Comparisons of Model Predictions to Measured Data. Radiat. Res. 164, 194-201 (2005).

7. R. K. Ratnayake, V. A. Semenenko and R. D. Stewart. Retrospective analysis of DSB rejoining data collected using warm-lysis PFGE protocols. Int. J Radiat. Biol. 81(6), 421428 (2005). Published erratum in Int. J Radiat. Biol. 82(2), 139 (2006)

8. V.A. Semenenko and R.D. Stewart. Fast Monte Carlo simulation of DNA damage formed by electrons and light ions. Phys. Med. Biol. 51(7), 1693-1706 (2006). 


\section{Computer Software}

As part of this project, several computer programs were developed and made freely available for educational, research and commercial purposes through the world wide web. They are

1. V.A. Semenenko and R.D. Stewart, Monte Carlo Damage Simulation (MCDS) Software. Version 2.01, March 2006. Available at http://rh.healthsciences.purdue.edu/mcds/

2. V.A. Semenenko and R.D. Stewart, Monte Carlo Excision Repair (MCER) Software. Version 2.01, March 2006. Available at http://rh.healthsciences.purdue.edu/mcer/

3. R.D. Stewart, Virtual Cell (VC) Radiobiology Software. PNNL-13579. Version 1.10J Released March 1, 2002. A beta of version 2.0 of the VC software was released in January 2005 and is available at http://rh.healthsciences.purdue.edu/vc/

\section{Presentations}

1. R.D. Stewart. Application of DNA damage and pathway-specific repair models to radiation mutagenesis. Workshop on Biologically-Based Modeling of Human Health Effects of Low Dose Ionizing Radiation. Fred Hutchinson Cancer Research Center, Seattle, WA July 28-29 (2005).

2. R.D. Stewart, V.A. Semenenko, and R.K. Ratnayake. Fast Monte Carlo Models to Simulate the Formation and Excision Repair of DNA Damage. Presented at the VIIth International Workshop on Radiation Damage to DNA, M. Weinfeld and D. Murray, Chair. Banff, Canada May 25-30, 2004.

3. R.D. Stewart, R.K. Ratnayake and V.A. Semenenko, DNA Repair and the Mutagenic and Cell Killing Potential of Singly and Multiply Damaged DNA Sites. Presented in the symposium Modeling and Experimental Approaches to Understanding DNA Damage, Repair and Their Consequences in Cell Biology, D.T. Goodhead, Chair. 51 ${ }^{\text {st }}$ Annual Meeting of the Radiation Research Society in St. Louis, MO, April 24 - 27, 2004.

4. R.D. Stewart, Can we predict radiation carcinogenesis from first principles? Presented at University of Michigan, Department of Nuclear Engineering. April 11, 2003.

5. R.D. Stewart, D.J. Carlson, and K. Jennings. The Virtual Cell and Multi-Endpoint Data Analysis. Presented at the 12th International Congress of Radiation Research (ICRR 2003), Brisbane Australia August 17-22 (2003).

6. H. Schöllnberger, R.D. Stewart, R.E.J. Mitchel, and W. Hofmann. An examination of radiation hormesis mechanisms using a multi-stage carcinogenesis model. Presented at the 12th International Congress of Radiation Research (ICRR 2003), Brisbane Australia August 17-22 (2003).

7. V.A. Semenenko and R.D. Stewart, Monte Carlo Simulation of the Base and Nucleotide Excision Repair of DNA Lesions. Presented at Lawrence Berkeley National Laboratory (July 22, 2003), University of California, Berkeley (July 23, 2003), Pacific Northwest National Laboratory (July 25, 2003).

8. R.D. Stewart, Can we predict radiation carcinogenesis from first principles? Invited Seminar. Presented at University of Michigan, Department of Nuclear Engineering. April 11, 2003.

9. RD Stewart, Hierarchical Modeling and the Virtual Cell Radiobiology Software. Invited Seminar. Department of Nuclear Engineering, North Carolina State University. May 9, 2002. 
10. H. Schollnberger, M.G. Ménache, R.D. Stewart, and W. Hofmann. Impact of cellular defense mechanisms and bystander effects on a multi-stage carcinogenesis model. International Conference on Non-Linear Dose-Response Relationships in Biology, Toxicology and Medicine, University of Massachusetts, Amherst, June 2002.

11. R.D. Stewart, E.J. Ackerman, X.C. Lei, B.R. Scott, and J.M. Malard. Hierarchical modeling of DNA repair, genomic instability, bystander effects, and the transformation or killing of cells. Presented at the $49^{\text {th }}$ Annual Meeting of the Radiation Research Society. Reno Hilton Hotel. Reno, Nevada, April 20-24, 2002.

12. R.D. Stewart, E.J. Ackerman, J.K. Shultis, and X.C. Lei. Modeling Bystander Effects Using a Microdosimetric Approach. Presented at the DOE Low Dose Radiation Research Program Workshop III. Rockville, Maryland, March 25-27, 2002. 